\title{
Proceso de atención de enfermería a un paciente con Parkinson más psicosis basado en las 14 necesidades de Virginia Henderson
}

\author{
María Salgado Quijano,* Guadalupe Espinosa Sánchez*
}

\begin{abstract}
RESUMEN
Hoy en día, la enfermería es considerada como una disciplina en el área de la salud, con gran impacto social que constantemente aumenta sus funciones para satisfacer las necesidades que impone el cuidado del paciente; en consecuencia, los cuidados van encaminados a ese mantenimiento de la vida, a través de la promoción de la higiene, la adecuada alimentación, el vestido y en general todas las medidas que hacen la vida más agradable. El cuidado va dirigido a mantener la salud de la persona en todas sus dimensiones: física, mental y social. La enfermera evalúa las necesidades de ayuda de la persona teniendo en cuenta sus percepciones y su globalidad.
\end{abstract}

Palabras clave: Cuidado, proceso de enfermería, Parkinson.

\section{Nursing process for a patient with Parkinson more psychosisbased on the 14 needs of Virginia Henderson}

\begin{abstract}
Today, nursing is seen as a discipline in the health area, with great social impact that continually raises its functions to meet the needs imposed by the health care, therefore care are aimed at the maintenance of life, through the promotion of hygiene, adequate food, clothing and in general all measures that make life more enjoyable. The care is aimed at maintaining the health of the person in all its dimensions, physical, mental and social. The nurse evaluates aid needs of the person taking into account their perceptions and whole.
\end{abstract}

Key words: Care, nursing process, Parkinson.

\section{JUSTIFICACIÓN}

$\mathrm{E}$ 1 interés de realizar el Proceso de Atención en Enfermería (PAE) en un paciente que presenta alteración de la necesidad de movilidad y postura secundaria a la enfermedad de Parkinson más psicosis es con el fin de llevar a cabo las acciones e intervenciones encaminadas a contribuir en los cuidados de la rehabilitación, así como aminorar las complicaciones de esta enfermedad en la persona; también a enseñar a sus familiares a comprender y aprender a brindar los cuidados necesarios, haciéndolos que participen en algunas actividades para que el paciente pueda valerse por sí mismo y sea independiente en su higiene y aliño. ${ }^{1}$
La Secretaría de Salud ha estimado en base a los datos obtenidos del Instituto Nacional de Neurología y Neurocirugía, que existen al menos 500,000 personas con enfermedad de Parkinson en el país. ${ }^{2}$

\section{OBJETIVOS}

\section{General}

- Brindar los cuidados de enfermería a personas con Parkinson más brote psicótico mejorando la calidad de vida de la persona.

* Licenciadas en Enfermería con curso postécnico de Psiquiatría.

Correspondencia: María Salgado Quijano. Insurgentes Sur Núm. 3877, Col. La Fama, Delegación Tlalpan, E-mail: msalgadoq@hotmail.com Este artículo puede ser consultado en versión completa en http://www.medigraphic.com/enfermerianeurologica 


\section{Específicos}

- Proporcionar cuidados holísticos, valorando las necesidades básicas de movilidad y postura.

- Elaborar diagnósticos de enfermería específicos en enfermos con Parkinson más brote psicótico.

\section{METODOLOGÍA DEL TRABAJO}

El procedimiento se lleva a cabo en el Instituto Nacional de Neurología y Neurocirugía en la Unidad de Neuropsiquiatría en un periodo que comprende del 14 al 25 de mayo de 2012 . La metodología implementada se apoya en el proceso enfermero ya que sistematiza la información a través de la valoración, diagnóstico, planeación, ejecución y evaluación; la información se obtiene utilizando la historia clínica.

\section{MARCO TEÓRICO}

\section{Proceso de Atención en Enfermería}

Es una herramienta indispensable en la ejecución de los cuidados de enfermería ya que se basa en ciertos pasos con ordenamiento lógico y estructurado que permiten la planeación y aplicación de destrezas intelectuales, interpersonales y técnicas de enfermería para ayudar a los pacientes a lograr su potencial máximo de salud.

La aplicación de la práctica asistencial enfermera es el método conocido como Proceso de Atención en Enfermería (PAE), el cual consta de cinco etapas, que son: ${ }^{3}$

\section{Valoración}

- Es la primera fase del proceso de enfermería que consiste en la recogida y organización de los datos que conciernen a la persona, familia y entorno

\section{Diagnóstico}

- Es el juicio o conclusión que procede como resultado de la valoración de la enfermería

\section{Planificación}

- Se desarrollan estrategias para prevenir, minimizar o corregir los problemas así como para promover la salud

\section{Ejecución}

- Es la realización o puesta en práctica de los cuidados programados

\section{Evaluación}

- Significa comparar las respuestas de la persona y determinar si se han conseguido los objetivos establecidos

\section{Teoría de Virginia Henderson en enfermería}

Virginia Henderson nació en 1897, originaria de Kansas City. Vivió una larga trayectoria profesional como autora e investigadora; entre sus múltiples publicaciones y participaciones en asociaciones y comités llegó a definir la enfermería en términos funcionales, lo que publicó en $1959 .{ }^{4}$ La teoría de Virginia Henderson es destacada, se basa en las necesidades humanas para la vida y la salud como núcleo para la actuación de enfermería, tiene como única función ayudar al individuo sano o enfermo en la realización de aquellas actividades que contribuyen a su salud o recuperación (o a una muerte tranquila).

\section{Supuestos principales de Henderson}

La enfermera tiene como única función ayudar a los enfermos y al equipo de salud a evaluar las necesidades básicas de la persona para mantener el equilibrio fisiológico y emocional y conseguir su independencia.

Las 14 necesidades básicas de la persona, según el modelo de Henderson son:

1. Respirar normalmente.

2. Comer y beber de forma adecuada.

3. Evacuar los desechos corporales.

4. Moverse y mantener una postura adecuada.

5. Dormir y descansar.

6. Elegir la ropa adecuada (para vestirse y desvestirse.

7. Mantener la temperatura del cuerpo dentro de un margen adecuado seleccionando la ropa y modificando las condiciones ambientales.

8. Mantener la higiene corporal y un buen aspecto y proteger la piel.

9. Evitar los peligros del entorno y daño a los demás.

10. Comunicarse con sus semejantes expresando las propias emociones, necesidades, temores u opiniones.

11. Actuar con arreglo a la propia fe.

12. Actuar de manera que se tenga la sensación de satisfacción con uno mismo.

13. Disfrutar o participar en diversas formas de entendimiento.

14. Aprender, descubrir o satisfacer la curiosidad para alcanzar un desarrollo y una salud normales y acudir a los centros sanitarios disponibles. ${ }^{5}$

\section{PRESENTACIÓN DEL CASO}

Se trata del Sr. JACC, masculino de 67 años de edad con antecedentes heredofamiliares (primos hermanos) con 
hipertensión arterial, sin datos de enfermedades crónicodegenerativas en la familia.

Inicia su padecimiento desde hace 15 años con diagnóstico de Parkinson. Actualmente presenta disminución de la fuerza en miembro torácico derecho, marcha claudicante y temblor fino en miembro pélvico derecho. El motivo de su ingreso al Servicio de Neuropsiquiatría es por presentar alucinaciones visuales consistentes "en hombres que entraban a su casa y que se dormían en medio de él y su esposa"; además pensaba que su esposa era la que propiciaba la entrada de estos hombres; por este motivo cierra puertas y se lleva a su cuarto un rifle de diábolos con el cual se podría, supuestamente, defender; también presenta insomnio y agresividad física y verbal heterodirigida, así como alteraciones de lenguaje. Se le inicia tratamiento a base de levodopa y benseracida (madopar) y biperideno (akineton). En el 2004 suspendió los fármacos e inició tratamiento con levodopa $50 \mathrm{mg} /$ carbidopa $12.5 \mathrm{mg}$ (stalevo), la cual ocasiona efectos adversos caracterizados por ansiedad e incontinencia urinaria, por lo que lo suspenden y se agrega pramipexol (sifrol), levodopa $250 \mathrm{mg} /$ carbidopa 25 $\mathrm{mg}$ (cloisone). También se le inicia tratamiento con quetiapina (seroquel) de $25 \mathrm{mg}$ para brote psicótico. Cabe mencionar que con el ajuste de medicamentos tuvo mejoría general importante. Se concluye con el ingreso del Sr. JACC al Servicio de Neuropsiquiatría con síntomas psicóticos secundarios al uso de medicamento, signos vitales de T/A: 110/70, FC: 70x', Fr: 20x', Temp: $36.2{ }^{\circ} \mathrm{C}$.

Se encuentra con temblor leve en miembro torácico derecho, con estado afectivo triste, aislado, con dificultad para la marcha y rigidez de hemicuerpo derecho, alucinaciones visuales, con ansiedad, demandante de atención, miedos e insomnio.

\section{JERARQUIZACIÓN DE NECESIDADES}

Necesidad

\section{Eliminación}

Moverse y mantener buena postura

Descanso y sueño

Uso de prendas de vestir adecuadas
Diagnóstico

Riesgo de incontinencia urinaria de urgencia relacionada por efectos secundarios a la ingesta de medicamento levodopa $50 \mathrm{mg} /$ carbidopa 12.5 $\mathrm{mg}$ (estalevo)

Deterioro de la movilidad física relacionada con déficit neuroquímico cerebral de dopamina, manifestado por afección progresiva de la movilidad e inestabilidad física, rigidez muscular y temblor involuntario

Trastorno del patrón de sueño relacionado con la terapia por efecto de medicamento (levodopa $50 \mathrm{mg} / \mathrm{carbido-}$ pa $12.5 \mathrm{mg}$ ) manifestado por dificultad para conciliar el sueño (insomnio) e irritabilidad

Déficit de autocuidado relacionado con movimientos incoordinados y deterioro de la capacidad para ponerse las prendas de vestir

Deterioro de la comunicación verbal relacionado con debilidad, rigidez de los músculos faciales; lenguaje lento y pausado

Confusión aguda relacionada con

Necesidad de comunicarse ideas delirantes de referencia y daño, manifestada por ver personas en su habitación que quieren lastimarlo Disminución de la agudeza visual de ojo derecho, manifestada por temor a la pérdida total de vista, así como a no realizar sus actividades cotidianas

Conocimientos deficientes sobre el tratamiento relacionado con falta

Necesidad de trabajar y realizarse de interés en el aprendizaje, manifestado por seguimiento inexacto de las instrucciones

Evitar peligros

Riesgo de lesiones relacionado con la inestabilidad postural y rigidez muscular

\section{PLAN DE CUIDADOS DE ENFERMERÍA}

\section{Diagnóstico de enfermería:}

Deterioro de la movilidad física relacionada con déficit neuroquímico cerebral de dopamina; afección progresiva de la movilidad e inestabilidad física, rigidez muscular y temblor involuntario.

Objetivo: Ayudar a mejorar la fuerza muscular, así como la coordinación y deambulación.

Intervención de enfermería persona, aplicando la regla de oro

Orientación acerca de la realización de ejercicios, valoración y programación de fisioterapia para la persona, que le ayuden a fortalecer la fuerza motora en brazos, manos y piernas

Motivarlo a que deambule, mencionándole la importancia que tiene el ejercitar sus músculos

Mencionarle cómo pueden disminuir los temblores finos de miembro superior derecho y rigidez de hemicuerpo derecho, cómo lograrlo y qué debe de hacer
Fundamentación científica

La levodopa es un medicamento que se trasforma en dopamina en el cerebro y sustituye su deficiencia, alivia la rigidez en la mayoría de las personas y suele mejorar el temblor algún tiempo; tiene vida media de 60 a 90 minutos

Los ejercicios ayudan a aumentar el vigor muscular, mejoran la coordinación y disminuyen la rigidez; con esto se evitan contracturas y deformidades óseas

Las técnicas aplicadas para caminar son con el fin de mejorar la marcha pesada y la tendencia a inclinarse hacia adelante, realizando pasos alargados con los pies separados; la marcha de pie ayudará al balanceo de brazos y hombros

El temblor fino disminuye con el reposo, aumenta con los movimientos bruscos y se intensifica con el estrés. El estrés puede precipitar un episodio agudo

Ejecución: Se valoró la fuerza muscular así como la movilidad de la persona durante su estancia hospitalaria; con el apoyo del familiar, se llevó a cabo la realización de ejercicios 
de flexión y extensión de manera programada de acuerdo a las necesidades de la persona y se ministra levodopa de acuerdo a las indicaciones médicas con hora exacta y dosis correcta, valorando los efectos secundarios.

Evaluación: Ha mejorado el tono muscular y fuerza, su deambulación es más coordinada, la ministración del medicamento ha contribuido a disminuir la rigidez de la persona y ésta reposa cuando es necesario.

Diagnóstico: Trastorno del patrón de sueño relacionado con la terapia por efecto de medicamento (levodopa) manifestado por dificultad para conciliar el sueño (insomnio) e irritabilidad. Objetivo: Lograr un patrón de sueño adecuado durante la noche.

Intervención de enfermería

Dar a conocer a la persona los efectos secundarios del medicamento que le está alterando su patrón del sueño

Disminuir los ruidos para proporcionar el menor número de molestias a la persona durante el periodo de sueño

Establecer un horario para ir a la cama así como proponerle a la persona una previa ducha, de preferencia durante la noche con agua tibia-caliente

Mantener ocupada a la persona durante el día participando activamente en las distintas actividades establecidas por el servicio durante su estancia hospitalaria como deambular, leer o participar en terapia ocupacional que contribuyan a mejorar su sueño

Fundamentación científica

Los efectos secundarios de la levodopa incluyen alteraciones como la incapacidad para conciliar el sueño, así como despertares nocturnos

Es importante favorecer un ambiente confortable y tranquilo para mejorar el descanso y un sueño completo

El baño completo de regadera o tina unos minutos antes de acostarse ayudará a un descanso pleno en la persona durante la noche y reducirá las tensiones nerviosas

La lectura, caminata y la participación en actividades manuales es una técnica que ayuda a la persona a permanecer ocupada durante el día, y mantenerla despierta es una forma de ayudarla a dormir plenamente por la noche

Ejecución: La modificación de la dosis del medicamento (levodopa), las técnicas de relajación y ejercicios, así como un ambiente tranquilo y libre de factores externos ayudaron a la persona a restablecer su hábito de sueño durante la noche. Evaluación: La persona ha incrementado sus horas de sueño nocturno y los efectos del medicamento han disminuido a partir de la modificación del mismo.

Diagnóstico: Deterioro de la comunicación verbal relacionada con debilidad, rigidez de los músculos faciales manifestada por hablar con lenguaje lento y pausado.

Objetivo: Ayudar a la persona a mejorar la comunicación.

Intervención de enfermería

Poner atención a la persona cuando hable y observar su expresión facial, gestos y tono de voz, que ayuden a identificar la manera de apoyarla

Demostrar interés y paciencia a la persona para proporcionarle confianza y que así exprese libremente sus sentimientos y temores
Pedir al fisioterapeuta su participación con terapias de lenguaje y ejercicios faciales (movimientos lengua, ejercicios faciales) que contribuyan a la rehabilitación de la persona y disminuyan sus limitaciones que afectan la comunicación

Orientar a la familia a fomentar la realización de los ejercicios por parte de la persona
La realización de ejercicios específicos ayudan a mejorar la comunicación de la persona; la terapia de ejercicios faciales contribuye a disminuir la rigidez de los músculos de la cara y mandíbula, lo que ayuda a mejorar la tonificación así como el lenguaje

La participación de la familia es fundamental para que la persona continúe con sus ejercicios durante su estancia hospitalaria, así como en su hogar al darlo de alta

Ejecución: Se valora el tono y volumen de la persona, así como la rigidez de los músculos faciales; se realizan terapias de lenguaje y se formulan preguntas sencillas que nos pueda contestar con un "sî" o un "no", y se le pide realizar lectura en voz alta. Evaluación: La persona mejora la comunicación y realiza los ejercicios indicados por el fisioterapeuta, por lo que su lenguaje es más fluido.

Diagnóstico: Confusión aguda relacionada con ideas delirantes de referencia y daño, manifestando ver personas en su habitación que quieren lastimarlo.

Objetivo: Disminuir los factores que le generen ansiedad y temores.

Intervención de enfermería

Valorar el estado perceptual de la persona para detectar lo que le ocasiona su ansiedad y temores

Evitar contrariar a la persona tratando de convencerlo de que sus ideas son falsas e irreales

Ubicar a la persona en la realidad con base en hechos reales y no insistir en las ideas delirantes

Proporcionar seguridad indicándole estamos cerca de él y sobre todo al pendiente

Ministración de la quetiapina, con la dosis prescrita por el médico, $25 \mathrm{mg}$, vía oral c/24 h. por la noche a reserva de algún ajuste del medicamento a la persona que no está sola, que

Fundamentación científica

La alteración de la percepción en el entorno de la persona puede generarle un estado de angustia que se manifieste en miedos y ansiedad

Saber cómo abordarlo sin que esto pueda generarle una conducta de irritabilidad, autoagresión y heteroagresión que ponga en peligro su persona o a otros

Reorientar a la persona en sus tres esferas (espacio, tiempo y persona), llamarlo por su nombre mencionándole la fecha actual y el lugar

La interrelación enfermera-paciente es importante para que la persona se sienta segura y tenga confianza y pueda expresar sus sentimientos $e$ inquietudes, y mejore su autoestima

La quetiapina es un antipsicótico que ayuda a la persona a disminuir sus ideas delirantes tales como: cambios en el comportamiento, pensamientos extraños y atemorizantes

Ejecución: La ministración adecuada del medicamento al paciente contribuyó a disminuir sus ideas de daño y referencia; se le brindó seguridad y confianza para que expresara con libertad sus sentimientos y temores.

Evaluación: La persona ha disminuido sus ideas delirantes de daño y alucinaciones visuales, así también la ansiedad y temores. La ministración del antipsicótico ha sido benéfica en el tratamiento. 


\section{CONCLUSIONES}

El proceso de atención de enfermería es un instrumento valioso para jerarquizar necesidades y brindar una atención oportuna, ayudando de esta manera a su pronta rehabilitación y/o recuperación. De tal manera que los objetivos planteados fueron cumplidos y se llevaron a cabo a través de la aplicación de actividades y cuidados encaminados a favorecer su calidad de vida y mantener el bienestar actual de la persona.

Es de vital importancia que el profesional de enfermería conozca no sólo el fin terapéutico de los medicamentos sino también sus efectos secundarios, ya que en este caso específico la sintomatología que la persona manifestaba era en gran parte provocada por reacciones adversas del medicamento.

Uno de los apoyos más relevantes fue el de familiares que contribuyeron durante la estancia hospitalaria con su participación afectiva a su familiar; esto fue un punto importante para su rehabilitación y su pronta recuperación e integración familiar.

Es importante mencionar que con la colaboración de la aplicación técnica de la fisioterapia mejoró la deambulación y la postura, ayudando a que las articulaciones y músculos no perdieran su movilidad y fuerza.

\section{ANEXO 1}

\section{Enfermedad de Parkinson}

La enfermedad de Parkinson es un trastorno neurodegenerativo crónico y progresivo y la segunda causa más común de incapacidad neurológica crónica; suele clasificarse como un trastorno del movimiento; también produce alteraciones en la función cognitiva, la expresión de emociones y la función autónoma. ${ }^{6}$

Los síntomas suelen iniciarse de una manera lenta y progresiva; a veces el primer síntoma es un estado depresivo, dificultad para girarse en la cama o para ponerse los zapatos. En otras circunstancias, son los familiares de la persona quienes notan que realiza todas las actividades un poco más lento de lo normal: más tiempo duchándose, al caminar, al escribir, etc.

La causa de la enfermedad de Parkinson sigue siendo oscura; pero es probable que se deba a muchos procesos patológicos diferentes con una intervención variable del envejecimiento, el ambiente y los factores genéticos. ${ }^{17}$

La esperanza de vida del paciente afectado por la enfermedad de Parkinson es igual al del resto de la población; sin embargo, la calidad de vida disminuye progresivamente a medida que la enfermedad avanza. La calidad de vida tam- bién depende de la manera en que se haya tratado al paciente. La enfermedad es un trastorno degenerativo de la llamada sustancia negra compacta (nigroestriado). Esta sustancia cerebral la forman neuronas secretoras del neurotransmisor dopamina (DA). El equilibrio, la postura, el tono muscular y el movimiento muscular involuntario dependen del equilibrio entre la producción de dos sustancias: DA (inhibidor) y acetilcolina (estimulador).

La acción de la dopamina sobre los ganglios basales es responsable de la fluidez de movimientos. Por tanto, la ausencia o muerte de estas neuronas secretoras DA implica que la acetilcolina tiene un efecto estimulador sin que exista el contrapunto de la DA.

Síntomas del "parkinsonismo": La persona presenta una pérdida progresiva de la habilidad psicomotriz. Todo esto resulta en muchas dificultades para satisfacer las necesidades básicas:

Temblor (en reposo): En las manos y la cabeza.

Rigidez muscular: Presenta dificultad para inclinarse, para doblar las articulaciones. Presenta una facies inexpresiva (jugador de poker) y dificultad para articular palabras. Hay dificultad para masticar y tragar (disfagia). Estos síntomas son más evidentes en fases más avanzadas de la enfermedad. Bradicinesia: Además de la rigidez muscular, los pacientes afectados de Parkinson tienen dificultad para iniciar el movimiento y controlar el movimiento fino (escritura, por ejemplo). Inestabilidad postural: Dificultad para mantener el equilibrio.

Manifestaciones típicas son la inexpresividad facial (cara del jugador de poker), la escasez de movimientos automáticos como el párpadeo o el braceo al caminar, la inclinación de tronco hacia delante durante la marcha.

Conforme aumenta la duración de la enfermedad pueden aparecer otros síntomas, como un deterioro de la marcha con dificultad fundamentalmente al inicio de ésta y en los giros, en los que el paciente se queda como pegado al suelo, fenómeno que se denomina imantación. También se puede producir una alteración de los reflejos de reequilibración dando lugar a caídas.

Tratamiento: Uno de los más utilizados y considerado de mayor eficacia es el siguiente:

Dopaminérgico: Los fármacos dopaminérgicos se utilizan para aumentar los niveles de DA en la zona del cerebro dañada. Utilizan unos mecanismos de acción:

Levodopa: Es el fármaco más utilizado y más eficaz contra el parkinsonismo. Es necesario administrarlo a grandes dosis porque sólo un $1 \%$ consigue atravesar la BHE; esto provoca que tenga gran cantidad de efectos secundarios. Las altas dosis suponen también un riesgo de toxicidad, por lo que estas personas deben ser monitorizadas para detectar síntomas de intoxicación. 
Por estos motivos, suele administrarse conjuntamente con otros fármacos que disminuyan estos efectos secundarios. Estos fármacos son la carbidopa (que impide que la levodopa se transforme en DA en sangre) y la doperidona (antagonista de la DA, por tanto, compite por los mismos receptores con la DA e impide que los estimule).

Ninguna de estas dos sustancias atraviesa la BHE, por lo que son fármacos que disminuyen los efectos secundarios de la levodopa a nivel periférico y no en el sistema nervioso central (SNC). ${ }^{8}$

\section{Psicosis en la enfermedad de Parkinson}

La psicosis se define como una deficiencia importante en la percepción de la realidad: los pacientes evalúan de manera incorrecta sus percepciones y obtienen inferencias inadecuadas acerca de la realidad exterior, lo cual conduce a delirio y alucinaciones. ${ }^{9}$

\section{Mente y personalidad del parkinsoniano}

En algunos pacientes es tan intenso el deterioro de las funciones mentales y psicológicas que llegan a representar un problema grave. En comparación con otras personas de su edad, presentan una serie de dificultades mentales o cognitivas de escasa intensidad. En algunos casos sólo al hacer determinados "test" o pruebas neuropsicológicas es cuando se pone de manifiesto el fallo. Las funciones que más se afectan son la integración visuoespacial y la forma en que se ejecutan determinadas acciones motoras. Esta disminución de la percepción e integración visuoespacial es la que les hace dudar cuando van a atravesar una puerta estrecha, y también puede contribuir a frecuentes caídas. El pensamiento del parkinsoniano es lento, carece de "reflejos mentales", su cerebro tarda mucho tiempo en procesar la información y resulta especialmente dificultoso cambiar de un programa motor a otro. Esto es lo que se conoce como bradifrenia, un síntoma que consiste en el enlentecimiento de determinados procesos mentales y que hay que diferenciar de la demencia. ${ }^{10}$

La conducta psicótica es muy común en la EP. Los principales síntomas son las alucinaciones visuales y el delirio paranoide. Estos síntomas inciden en la calidad de vida tanto del paciente como de su cuidador.

Puede ser causada por una combinación de factores endógenos y exógenos que afectan varios sistemas. Se considera que los factores endógenos más importantes son la denervación mesocortical dopaminérgica, la denervación colinérgica neocortical y las alteraciones del sueño MOR inducidas por los cambios colinérgicos, las cuales agravan el estado de confusión endógeno. Por otra parte, factores exógenos importantes son los tratamientos crónicos con dopaminomiméticos (psicosis subaguda) o anticolinérgicos (confusión tóxica), así como otros factores psicogénicos como edad, deshidratación, infecciones, abstinencia de alcohol o de drogas, fiebre, aislamiento o sobrecarga social, los cuales conducen a psicosis exógenas.

\section{BIBLIOGRAFÍA}

1. Kerouac S. Pensamiento enfermero, 1996, $1^{a}$ edición en español, Editorial Elsevier Masson, Barcelona España. 10.

2. http://www.ssm.gob.mx/pdf/salud_mental/guias_tecnicas/parkinson. pdf p.p. 24 fecha: 25-06-2012 hora: 23:06.

3. Marriner A. Proceso de atención de enfermería, un enfoque científico. $2^{\mathrm{a}}$ edición, Editorial El Manual Moderno, S.A de C.V., México, D.F. 1983: 1-4.

4. Marriner TA. Modelos y teorías de enfermería, 1997, $4^{a}$ edición en español, Editorial, Harcourt Brace, Madrid España; 99, 100.

5. Marriner TA. Modelos y teorías de enfermería, 1997, $4^{\text {a }}$ edición en español, Editorial, Harcourt Brace, Madrid España; 102.

6. Stokes M. Fisioterapia en la rehabilitación neurológica, 2006, $2^{\text {a }}$ Edición en Español, Editorial, Elsevier Mosby, Madrid, España, 221.

7. Stokes M. Fisioterapia en la rehabilitación neurológica, 2006, $2^{a}$ Edición en Español, Editorial, Elsevier Mosby, Madrid, España, 222.

8. http://ocw.uib.es/ocw/infermeria/farmacologia/m3-tema2 p.p 5 fecha 26-06-2012 hora 20:17

9. http://www.scielo.org.mx/scielo.php? pid=S 0187 47052004000100006\&script=sci_arttext párrafo. 5 fecha 26-06-2012 hora 14:20

10. Micheli FE. Enfermedad de Parkinson y trastornos relacionados. Ed. Médica Panamericana. Buenos Aires, 1998: 342-359. 